\title{
MAGNETIC STEPS ON THE THRESHOLD OF THE NORMAL STATE
}

\author{
WAFAA ASSAAD
}

\begin{abstract}
Superconductivity in the presence of a step magnetic field has been recently the focus of many works. This contribution examines the behavior of a two-dimensional superconducting domain, when superconductivity is lost in the whole domain except near the intersection points of the discontinuity edge and the boundary. The problem involves its own effective energy. We provide local estimates of the minimizers in neighbourhoods of the intersection points. Consequently, we introduce new critical fields marking the loss of superconductivity in the vicinity of these points. The study is modelled by the Ginzburg-Landau theory, and large Ginzburg-Landau parameters are considered.
\end{abstract}

Keywords: Ginzburg-Landau functional, magnetic Schrödinger operators, superconductivity, step magnetic fields.

MSC subject classification: 35Q56, 35J10, 35P15.

\section{INTRODUCTION}

Hundreds of contributions have investigated the response of a type-II superconductor with a large Ginzburg-Landau (GL) parameter to applied magnetic fields (see the two monographs [SS07, FH10] in the mathematical literature). In many generic situations in two- and three-dimensional domains submitted to smooth magnetic fields, it is shown that superconductivity eventually breaks down under an increasing magnetic field [SJG63, GP99, LP00, HM01, HP03, FH10]; the superconductor is said to pass to the normal state. In these situations, the transition to the normal state occurs at a unique value of the applied field's intensity ${ }^{1}$, called the third critical field and depending on the GL parameter. The last phase preceding such a transition has been extensively studied for two-dimensional domains with piecewise smooth boundary (having possibly a finite number of corners) submitted to smooth magnetic fields (see e.g. [Jad01, Pan02, PK02, Bon05, BND06, BNF07, FH10, HK18, CG19]).

Here, we study the aforementioned phase in the case of a certain discontinuous magnetic field. In this situation, we provide precise information about the superconductivity localization, right before its breakdown. Such a localization was suggested but not proven in the recent paper [Ass20].

1.1. The functional. The problem is modelled by the GL theory. We consider a cross section, $\Omega \subset \mathbb{R}^{2}$, of an infinite cylindrical wire subjected to a magnetic field, whose direction is parallel to the axis of the cylinder and whose profile (the scalar magnetic field) is the function $B_{0} \in L^{2}(\Omega ;[-1,1])$. $\Omega$ is assumed to be open, bounded with a smooth boundary, and simply connected. The GL free energy is given by the functional:

$\mathcal{E}_{\kappa, H}(\psi, \mathbf{A})=\int_{\Omega}\left(|(\nabla-i \kappa H \mathbf{A}) \psi|^{2}-\kappa^{2}|\psi|^{2}+\frac{\kappa^{2}}{2}|\psi|^{4}\right) d x+\kappa^{2} H^{2} \int_{\Omega}\left|\operatorname{curl} \mathbf{A}-B_{0}\right|^{2} d x$,

\footnotetext{
${ }^{1}$ We say that the transition is monotone. For counterexamples of such a monotonicity, see e.g. [LP62, Erd97, FPS15, HK19, KPS19, KP19].
} 
with $\psi \in H^{1}(\Omega ; \mathbb{C})$ and $\mathbf{A} \in H^{1}\left(\Omega ; \mathbb{R}^{2}\right) . \psi$ is the order parameter with $|\psi|^{2}$ being a measure of the Cooper pair electrons density, and $\mathbf{A}$ is the vector potential whose curl represents the induced magnetic field in the sample. $\kappa>0$ is a characteristic scale of the sample called the GL parameter, assumed to be large $(\kappa \rightarrow+\infty)$, which corresponds to extreme type-II superconductors in physics. Finally, $H>0$ is the intensity of the applied magnetic field.

The functional in (1.1) admits a gauge invariance property ${ }^{2}$. Hence, one may restrict the energy minimization with respect to $(\psi, \mathbf{A})$ (originally done in the space $\left.H^{1}(\Omega ; \mathbb{C}) \times H^{1}\left(\Omega ; \mathbb{R}^{2}\right)\right)$ to the space $H^{1}(\Omega ; \mathbb{C}) \times H_{\text {div }}^{1}(\Omega)$, where

$$
H_{\text {div }}^{1}(\Omega)=\left\{\mathbf{A} \in H^{1}\left(\Omega ; \mathbb{R}^{2}\right): \operatorname{div} \mathbf{A}=0 \text { in } \Omega, \mathbf{A} \cdot \nu=0 \text { on } \partial \Omega\right\}
$$

and $\nu$ is a unit normal vector of $\partial \Omega$. We define the following ground-state energy

$$
\mathrm{E}_{\text {g.st }}(\kappa, H)=\inf \left\{\mathcal{E}_{\kappa, H}(\psi, \mathbf{A}):(\psi, \mathbf{A}) \in H^{1}(\Omega ; \mathbb{C}) \times H_{\text {div }}^{1}(\Omega)\right\} .
$$

Critical points $(\psi, \mathbf{A}) \in H^{1}(\Omega ; \mathbb{C}) \times H_{\text {div }}^{1}(\Omega)$ of $\mathcal{E}_{\kappa, H}$ are weak solutions of the following GL equations:

$$
\begin{cases}(\nabla-i \kappa H \mathbf{A})^{2} \psi=\kappa^{2}\left(|\psi|^{2}-1\right) \psi & \text { in } \Omega, \\ -\nabla^{\perp}\left(\operatorname{curl} \mathbf{A}-B_{0}\right)=\frac{1}{\kappa H} \operatorname{Im}(\bar{\psi}(\nabla-i \kappa H \mathbf{A}) \psi) & \text { in } \Omega, \\ \nu \cdot(\nabla-i \kappa H \mathbf{A}) \psi=0 & \text { on } \partial \Omega, \\ \operatorname{curl} \mathbf{A}=B_{0} & \text { on } \partial \Omega,\end{cases}
$$

with $\nabla^{\perp}=\left(\partial_{x_{2}},-\partial_{x_{1}}\right)$.

1.2. Literature summary and new contribution. The scenarios occurring in the case of smooth applied magnetic fields are well-known in the literature (see e.g. [LP99, HM01, PK02, SS07, Ray09, FH10, FK11, HK15, Att15a, Att15b, CR16a, CR16b, CG17, DMR18, FMP19]).

In particular, in domains with smooth boundaries submitted to uniform magnetic fields, three subsequent transitions are observed while increasing the intensity of the applied field, and correspondingly three critical values of the field's intensity - critical fields - are considered:

- The first critical field $H_{C_{1}}^{\text {unif }}(\kappa)=\mathcal{O}\left(\kappa^{-1} \log (\kappa)\right)$ : indicating the transition between the perfect superconductivity state, where the whole sample is superconducting, and the state of vortices nucleation.

- The second critical field $H_{C_{2}}^{\text {unif }}(\kappa)=\kappa$ : indicating the transition between the bulk and surface superconductivity states. In the surface state, superconductivity is exclusively and uniformly distributed along the boundary.

- The third critical field $H_{C_{3}}^{\text {unif }}(\kappa)=\mathcal{O}\left(\Theta_{0}^{-1} \kappa\right)$, where $\Theta_{0} \in(0,1)$ is introduced later: indicating the transition between the surface and normal states.

This picture is modified if the boundary of the sample admits singularities, like corners. In such situations, when the magnetic field is uniform, the bulk phase is not essentially altered, but the surface and normal phases prove to be affected by the presence of corners [Jad01, Pan02, BNF07, CG17, HK18, CG19]. In particular, under a certain spectral/geometric assumption (see [BNF07, Remark 1.1 \& Assumption 1.3]), the third critical field experiences a shift up in the presence of corners $\left(H_{C_{3}}^{\text {cor }}(\kappa)>H_{C_{3}}^{\text {unif }}(\kappa)\right)$, although is still of order $\kappa$. In addition, a new subphase appears in the surface superconductivity phase, when $H$ exceeds a certain critical value $H_{\text {int }}^{\text {cor }}(\kappa)$. There, superconductivity is no longer uniformly distributed

\footnotetext{
${ }^{2}$ The physically relevant quantities $|\psi|^{2}$, curl $\mathbf{A}$ and $|(\nabla-i \kappa H \mathbf{A}) \psi|^{2}$ are invariant under the transformation $(\psi, \mathbf{A}) \mapsto\left(e^{i \varphi \kappa H} \psi, \mathbf{A}+\nabla \varphi\right)$ for any $\varphi \in H^{2}(\Omega ; \mathbb{R})$.
} 
along the boundary, but solely localized at the corners. Moreover, superconductivity disappears at a corner, $\mathbf{s}_{j}$, once the field's intensity exceeds certain critical field $H_{j}^{\text {cor }}(\kappa) \in\left(H_{\mathrm{int}}^{\mathrm{cor}}(\kappa), H_{C_{3}}^{\mathrm{cor}}(\kappa)\right)$, depending on the opening angle of this corner (see e.g. [BNF07, HK18]).

Discontinuous magnetic fields were treated for the first time in the context of the non-linear GL functional in (1.1), in the recent works [AK16, AKPS19, Ass20]. These works considered a magnetic field $B_{0}$ which is a step function, satisfying the following assumptions (see Figure 1):

\section{Assumption 1.1.}

(1) $\Omega_{1}$ and $\Omega_{2}$ are two disjoint open sets.

(2) $\Omega_{1}$ and $\Omega_{2}$ have a finite number of connected components.

(3) $\partial \Omega_{1}$ and $\partial \Omega_{2}$ are piecewise smooth with a finite number of corners.

(4) $\Gamma=\partial \Omega_{1} \cap \partial \Omega_{2}$ is the union of a finite number of disjoint simple smooth curves $\left\{\Gamma_{k}\right\}_{k \in \mathcal{K}}$; we will refer to $\Gamma$ as the magnetic edge.

(5) $\Omega=\left(\Omega_{1} \cup \Omega_{2} \cup \Gamma\right)^{\circ}$ and $\partial \Omega$ is smooth.

(6) For any $k \in \mathcal{K}, \Gamma_{k}$ intersects $\partial \Omega$ at two distinct points. This intersection is transversal, i.e. $\mathrm{T}_{\partial \Omega} \times \mathrm{T}_{\Gamma_{k}} \neq 0$ at the intersection point, where $\mathrm{T}_{\partial \Omega}$ and $\mathrm{T}_{\Gamma_{k}}$ are respectively unit tangent vectors of $\partial \Omega$ and $\Gamma_{k}$.

(7) $B_{0}=\mathbb{1}_{\Omega_{1}}+a \mathbb{1}_{\Omega_{2}}$, where $a \in[-1,1) \backslash\{0\}$ is a given constant.

We borrow the following notation from [Ass20]:

Notation 1.2. Since $\Gamma \cap \partial \Omega$ is finite, we denote by

$$
\Gamma \cap \partial \Omega=\left\{\mathbf{p}_{j}: j \in\{1, \cdots, n\}\right\},
$$

where $n=\operatorname{Card}(\Gamma \cap \partial \Omega)$. For all $j \in\{1, \cdots, n\}$, let $\alpha_{j} \in(0, \pi)$ be the angle between $\Gamma$ and $\partial \Omega$ at the intersection point $\mathrm{p}_{j}$ (measured towards $\Omega_{1}$ ).
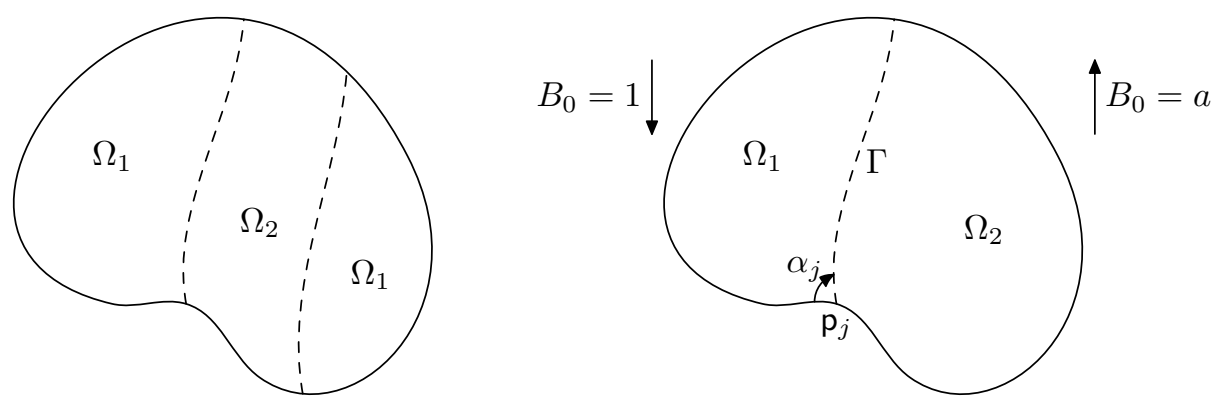

Figure 1. Schematic representation of the set $\Omega$ subjected to the step magnetic field $B_{0}$, with the magnetic edge $\Gamma$.

The study of such a discontinuous field case has been mathematically and physically motivated in [AK16, AKPS19, Ass20], and more recently in [AKS20]. The latter contribution established the well-posedness of such problems with step magnetic fields, in the sense that the study outcomes are not essentially affected by a small perturbation of the field. More precisely, it showed that the ground-state energy in (1.2) with the step magnetic field is the limit of ground-state energies with smooth fields. This interestingly constructed a bridge between the non-linear GL problems with discontinuous fields, tackled lately, and those with smooth fields, broadly studied in the literature as mentioned earlier.

[AK16, AKPS19, Ass20] examined superconductivity along an intensity interval extended from the bulk regime to the normal regime. Working under certain spectral conditions (Assumption 1.4 below), these three papers introduced the following critical fields: 
- $H_{C_{2}}^{\text {step }}(\kappa)=|a|^{-1} \kappa$ ( $a$ is the value in Assumption 1.1): marking the passage from bulk superconductivity to superconductivity being partially/globally distributed along $\Gamma \cup \partial \Omega$ (see [AKPS19, Section 1.5]).

- $H_{\mathrm{int}}^{\text {step }}(\kappa)=\left(|a| \Theta_{0}\right)^{-1} \kappa$ : marking the disappearance of superconductivity in the whole sample away from $\Gamma \cap \partial \Omega$.

- $H_{C_{3}}^{\text {step }}(\kappa)=\mathcal{O}\left(\mu_{*}^{-1} \kappa\right)$, for a certain $\mu_{*}<|a| \Theta_{0}$ introduced later: marking the passage to the normal state.

In particular, [Ass20] revealed a remarkable similarity between the role of $\Gamma \cap \partial \Omega$, and that of the corners in the foregoing corners situation. Indeed, $\Gamma \cap \partial \Omega$ shifts the third critical field to a higher level, compared to that for smooth domains with uniform fields $\left(H_{C_{3}}^{\text {step }}(\kappa)>H_{C_{3}}^{\text {unif }}(\kappa)\right)$. Moreover, a spectral value, $\mu\left(\alpha_{j}, a\right)$, was assigned to each intersection point $\mathrm{p}_{j}$ of $\Gamma$ and $\partial \Omega$. Then under the condition $\mu\left(\alpha_{j}, a\right)<|a| \Theta_{0}$ (discussed later), [Ass20] introduced additional fields, $H_{j}^{\text {step }}(\kappa):=\left(\mu\left(\alpha_{j}, a\right)\right)^{-1} \kappa$, satisfying $H_{\mathrm{int}}^{\text {step }}(\kappa)<H_{j}^{\text {step }}(\kappa)<H_{C_{3}}^{\text {step }}(\kappa)$. When $H>H_{j}^{\text {step }}(\kappa)$, [Ass20, Theorem 1.6] showed the non-existence of superconductivity near the point $\mathrm{p}_{j}$. This was reminiscent of the aforementioned corners performance (see [Ass20, Section 1.3] for a more detailed comparison). However, this theorem did not ensure the existence of superconductivity in the vicinity of $\mathrm{p}_{j}$, when $H$ is still below $H_{j}^{\text {step }}(\kappa)$. If such an existence is proven, then $H_{j}^{\text {step }}(\kappa)$ can be reintroduced as a critical field denoting the ending of the superconducting state near $\mathrm{p}_{j}$. This will emphasize the similarity with the corners situation in [BNF07, HK18].

Working under the assumptions in [Ass20], the current contribution establishes this existence, hence sharpens the results in [Ass20, Theorem 1.6]. More precisely, Theorem 1.5 below provides local estimates of the minimizers which describe the sample's behavior at the threshold of $H_{j}^{\text {step }}(\kappa)$, and particularly show the concentration of superconductivity near the point $\mathrm{p}_{j}$ when $H<H_{j}^{\text {step }}(\kappa)$.

1.3. Setting and main results. In what follows, we formally present the setting and the main results of this paper. In addition to Assumption 1.1, we assume the following:

Assumption 1.3. The intensity $H$ depends on $\kappa$ in the following manner

$$
H=b \kappa,
$$

where $b$ is a real parameter independent of $H$ and $\kappa$, satisfying

$$
b>\frac{1}{|a| \Theta_{0}} .
$$

Assumption 1.4. For $j \in\{1, \cdots, n\}$, let $\alpha_{j}$ be the angle in Notation 1.2. We assume that $\mu\left(\alpha_{j}, a\right)<|a| \Theta_{0}$.

The values $\Theta_{0}$ and $\mu\left(\alpha_{j}, a\right)$ appearing in the assumptions above stand for the following quantities:

- $\Theta_{0} \approx 0.59$ is the so-called de Gennes constant-the ground-state energy of the Neumann realization of the Schrödinger operator, $-\left(\nabla+\frac{1}{2} i x^{\perp}\right)^{2}$, with a unit magnetic field in $\mathbb{R}_{+}^{2}$ (see e.g. [BNF07]).

- $\mu(\alpha, a)$ is the ground-state energy of the Neumann realization of a Schrödinger operator with a step magnetic field in $\mathbb{R}_{+}^{2}$, introduced in Section 2.

Remark. The conditions in Assumption 1.4 are discussed later in the paper (see Remark 2.1 and Footnote 5). [Ass20, Section 3.3] provided examples of couples $\left(\alpha_{j}, a\right)$ satisfying this assumption, living in a neighbourhood of $(\pi / 2,-1)$. 
The statements of our main results involve the function

$$
E_{\alpha, a}:\left[\left(|a| \Theta_{0}\right)^{-1},+\infty\right) \rightarrow(-\infty, 0]
$$

defined in Section 3. $E_{\alpha, a}$ is a new effective energy constructed in this paper to deal with our problem near the intersection of the magnetic edge with the boundary. We highlight the following crucial property of this energy:

$$
E_{\alpha, a}(b)<0, \text { for } \frac{1}{|a| \Theta_{0}}<b<\frac{1}{\mu(\alpha, a)}, \text { and } E_{\alpha, a}(b)=0, \text { for } b \geq \frac{1}{\mu(\alpha, a)} .
$$

Let $j \in\{1, \cdots, n\}$ and $\ell \in(0,1)$. For each $\mathrm{p}_{j} \in \Gamma \cap \partial \Omega$, we define the set

$$
\mathcal{N}_{j}(\ell)=\left\{x \in \Omega, \operatorname{dist}\left(x, \mathrm{p}_{j}\right) \leq \ell\right\}
$$

We also introduce the set

$$
T=\left\{j \in\{1, \cdots, n\}: \mu\left(\alpha_{j}, a\right)<b^{-1}\right\}
$$

Theorem 1.5. Let $\rho \in(4 / 5,1)$. There exists $\kappa_{0}>1$ and a function $\mathfrak{r}:\left(\kappa_{0},+\infty\right) \rightarrow$ $(0,+\infty)$ such that $\lim _{\kappa \rightarrow+\infty} \mathfrak{r}(\kappa)=0$ and the following is true. If $\kappa \geq \kappa_{0}$ and $(\psi, \mathbf{A}) \in H^{1}(\Omega ; \mathbb{C}) \times H_{\mathrm{div}}^{1}(\Omega)$ is a minimizer of $(1.1)$, then for $\ell \approx \kappa^{-\rho}$ and $j \in T$ we have

$$
\left.\left|\kappa^{2} \int_{\mathcal{N}_{j}(\ell)}\right| \psi\right|^{4} d x+2 E_{\alpha_{j}, a}(b) \mid \leq \mathfrak{r}(\kappa)
$$

Consequently,

$$
\mathrm{E}_{\mathrm{g} . \mathrm{st}}(\kappa, H)=\sum_{j \in T} E_{\alpha_{j}, a}(b)+o(1) .
$$

The results in Theorem 1.5 are actually valid for any $j \in\{1, \cdots, n\}$ (not only for $j \in T)$. However in light of the properties of the energies $E_{\alpha_{j}, a}$ in (1.4), for a fixed intensity $H=b \kappa$ (a fixed $b$ ), superconductivity is negligible at the points $\left\{\mathrm{p}_{j}\right\}_{j \notin T}$. Hence, the only intersection points which contribute to the global energy, $\mathrm{E}_{\mathrm{g} . \mathrm{st}}(\kappa, H)$, are $\left\{\mathbf{p}_{j}\right\}_{j \in T}$.

On the other hand, when we increase the intensity $H$ (increasing $b$ ), we observe successive breakdowns of superconductivity at the points $\mathrm{p}_{j}$ : Labelling the points $\mathrm{p}_{j}, j \in\{1, \cdots, n\}$, so that

$$
\mu\left(\alpha_{1}, a\right) \geq \mu\left(\alpha_{2}, a\right) \geq \cdots \geq \mu\left(\alpha_{n}, a\right)
$$

the fields $H_{j}^{\text {step }}(\kappa):=\left(\mu\left(\alpha_{j}, a\right)\right)^{-1} \kappa$, introduced above, satisfy

$$
H_{C_{2}}^{\text {step }}(\kappa)<H_{\text {int }}^{\text {step }}(\kappa)<H_{1}^{\text {step }}(\kappa) \leq H_{2}^{\text {step }}(\kappa) \leq \cdots \leq H_{n}^{\text {step }}(\kappa) .
$$

By [Ass20], $H_{n}^{\text {step }}(\kappa)$ is the leading-order term of the third critical field ${ }^{3} H_{C_{3}}^{\text {step }}(\kappa)$. Thanks to Theorem 1.5, we now consider each field $H_{j}^{\text {step }}(\kappa)$ as a new critical field at which superconductivity disappears near the intersection point $\mathrm{p}_{j}$.

Consequently, this paper adds interesting features to the results in [AK16,AKPS19, Ass20] that we summarize in Diagram 2.

\footnotetext{
${ }^{3}$ The value $\mu_{*}$ mentioned earlier is then $\mu\left(\alpha_{n}, a\right)=\min _{j} \mu\left(\alpha_{j}, a\right)$.
} 


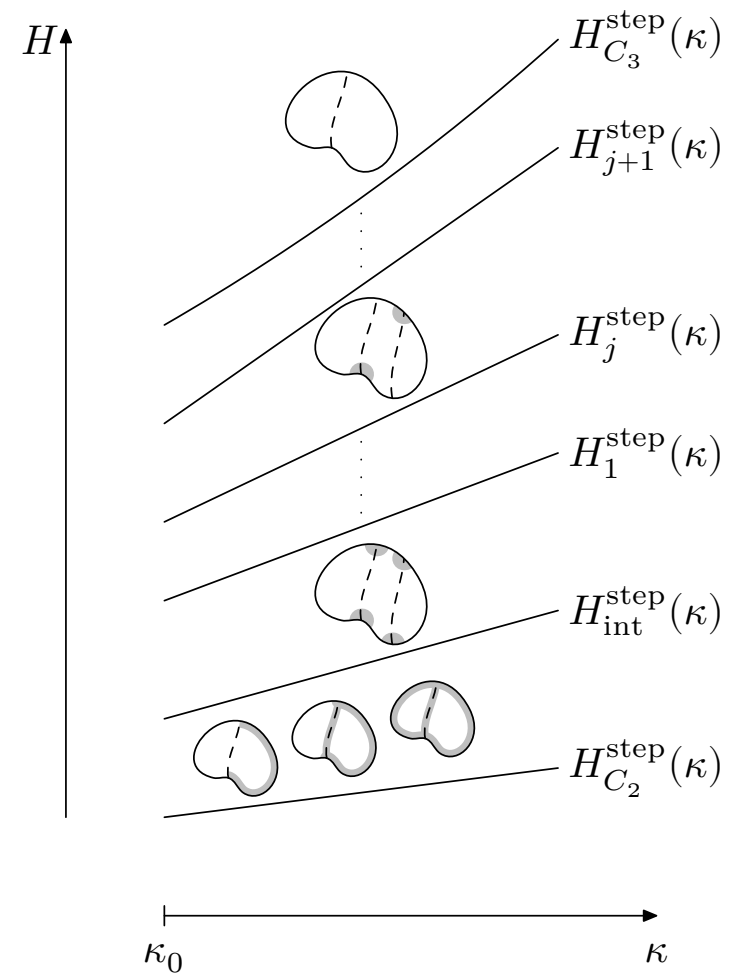

Figure 2. Schematic phase-diagram representing the distribution of superconductivity in the sample, according to the intensity $H$ of the applied magnetic field and for large values of $\kappa$. Only the grey regions carry superconductivity. The critical lines plotted in the $(\kappa, H)$-plane represent the critical fields in (1.8). When (and if) $H_{j}^{\text {step }}(\kappa)<H<$ $H_{j+1}^{\text {step }}(\kappa)$, superconductivity only persists near the points $\mathrm{p}_{k}$, for $k \geq j+1$.

1.4. Perspectives. The paper shows that superconductivity is mainly dictated by the intersection of $\Gamma$ and $\partial \Omega$, in the intensity regime $\left(H_{\text {int }}^{\text {step }}(\kappa), H_{C_{3}}^{\text {step }}(\kappa)\right)$ preceding the transition to the normal state. Note that this intersection effects do not show up in the leading-order terms of the energy, in the regime $\left(H_{C_{2}}^{\text {step }}(\kappa), H_{\text {int }}^{\text {step }}(\kappa)\right)$ where superconductivity is concentrated near $\Gamma \cup \partial \Omega$. In [AKPS19, Theorem 1.7], the following estimates of the ground-state energy are established, for $H \in\left(H_{C_{2}}^{\text {step }}(\kappa), H_{\mathrm{int}}^{\text {step }}(\kappa)\right)$ :

$$
\mathrm{E}_{\mathrm{g} . \mathrm{st}}(\kappa, H)=E_{a}^{\mathrm{L}}(b) \kappa+o(\kappa) \quad(\kappa \rightarrow+\infty),
$$

where $b=H / \kappa$ and $E_{a}^{\mathrm{L}}(b)$ is a $1 \mathrm{D}$ energy corresponding to the contribution of $\Gamma$ and $\partial \Omega$ away from their intersection.

However, predicting a similarity with the corners situation in the surface superconductivity regime, we expect the contribution of $\Gamma \cap \partial \Omega$ to the remainder terms in (1.9) as follows. [CG17, CG19] (see also [CR14, CR16a, CDR17]) have examined the effects of corners on surface superconductivity, in a 2D domain submitted to a uniform field. Inspired by these works, one may identify an effective model with a step magnetic field on the half-plane, revealing the foregoing contribution of $\Gamma \cap \partial \Omega$. This model will be genuinely $2 \mathrm{D}$, unlike the model generating the $1 \mathrm{D}$ energy $E_{a}^{\mathrm{L}}(b)$.

Analogously to [CG19, Theorem 2.1], we anticipate the following improved expansion in (1.9):

$$
E_{a}^{\mathrm{L}}(b) \kappa-E_{\mathrm{corr}}^{1} \int_{0}^{\partial \Omega_{1}} k(s) d s-E_{\mathrm{corr}}^{2} \int_{0}^{\partial \Omega_{2}} k(s) d s-E_{\mathrm{corr}}^{3} \int_{0}^{\Gamma} k(s) d s-\sum_{j=1}^{N} E_{\mathrm{int}, \alpha_{j}, a}+o(1),
$$


where $E_{\text {corr }}^{k}, k=1, \cdots, 3$, are correction energies corresponding to the curvature contribution along $\Gamma$ and $\partial \Omega$, and $E_{\text {int }, \alpha_{j}, a}, j=1, \cdots, N$, are energies corresponding to the contributions of the intersection points $\mathrm{p}_{j}$, and depending on the angles $\alpha_{j}$ and the values $a$ of the applied magnetic field.

\subsection{Notation.}

- Let $a(\kappa)$ and $b(\kappa)$ be two positive functions. We write $a(\kappa) \approx b(\kappa)$ if there exist constants $\kappa_{0}, C_{1}$ and $C_{2}$ such that for all $\kappa \geq \kappa_{0}, C_{1} a(\kappa) \leq b(\kappa) \leq$ $C_{2} a(\kappa)$. We write $a(\kappa)=\mathcal{O}(b(\kappa))$ if $a(\kappa) / b(\kappa) \rightarrow 1$ as $\kappa \rightarrow+\infty$.

- The quantity $o(1)$ indicates a function of $\kappa$ such that $|o(1)| \rightarrow 0$ as $\kappa \rightarrow+\infty$. Any expression $o(1)$ is independent of the minimizer $(\psi, \mathbf{A})$ of $(1.1)$.

- Let $n \in \mathbb{N}$ and $N \in \mathbb{N}, \gamma \in(0,1)$. We use the following Hölder space

$$
C^{n, \gamma}(\bar{\Omega})=\left\{f \in C^{n}(\bar{\Omega}) \mid \sup _{x \neq y \in \Omega} \frac{\left|D^{n} f(x)-D^{n} f(y)\right|}{|x-y|^{\alpha}}<+\infty\right\} .
$$

1.6. Organization of the paper. Sections 2 and 3 present the effective energies and their properties in the half-plane. Section 4 gathers useful a-priori estimates and decay results. In Section 5, a suitable change of variables is defined and the local estimates in Theorem 1.5 are established.

\section{Shrödinger OPERATOR With A SteP MAGNeTIC FIELD IN THE HALF-PLANE}

In this section, we present a Schrödinger operator with a step magnetic field in $\mathbb{R}_{+}^{2}$, introduced in [Ass20] and whose spectral properties are involved in the study of the effective energy in Section 3. The spectral analysis of this operator was first done in [Ass20].

Let $a \in[-1,1) \backslash\{0\}$ and $\alpha \in(0, \pi)$. We introduce the following sets in polar coordinates

$$
\begin{aligned}
& D_{\alpha}^{1}=\left\{r(\cos \theta, \sin \theta) \in \mathbb{R}^{2}: r \in(0, \infty), 0<\theta<\alpha\right\}, \\
& D_{\alpha}^{2}=\left\{r(\cos \theta, \sin \theta) \in \mathbb{R}^{2}: r \in(0, \infty), \alpha<\theta<\pi\right\},
\end{aligned}
$$

and the Schrödinger operator in $\mathbb{R}_{+}^{2}$

$$
\mathcal{H}_{\alpha, a}=-\left(\nabla-i \mathbf{A}_{\alpha, a}\right)^{2},
$$

where $\mathbf{A}_{\alpha, a}=\left(0, A_{\alpha, a}\right)$ is a magnetic potential ${ }^{4}$ defined as follows:

$$
\begin{aligned}
\text { For } \alpha \in(0, \pi / 2), \quad A_{\alpha, a}\left(x_{1}, x_{2}\right) & = \begin{cases}x_{1}+\frac{a-1}{\tan \alpha} x_{2}, & \text { if }\left(x_{1}, x_{2}\right) \in D_{\alpha}^{1}, \\
a x_{1}, & \text { if }\left(x_{1}, x_{2}\right) \in D_{\alpha}^{2},\end{cases} \\
\text { for } \alpha \in(\pi / 2, \pi), \quad A_{\alpha, a}\left(x_{1}, x_{2}\right) & = \begin{cases}x_{1}, & \text { if }\left(x_{1}, x_{2}\right) \in D_{\alpha}^{1}, \\
a x_{1}+\frac{1-a}{\tan \alpha} x_{2}, & \text { if }\left(x_{1}, x_{2}\right) \in D_{\alpha}^{2},\end{cases} \\
\text { and } \quad A_{\frac{\pi}{2}, a}\left(x_{1}, x_{2}\right) & = \begin{cases}x_{1}, & \text { if }\left(x_{1}, x_{2}\right) \in D_{\pi / 2}^{1}, \\
a x_{1}, & \text { if }\left(x_{1}, x_{2}\right) \in D_{\pi / 2}^{2} .\end{cases}
\end{aligned}
$$

\footnotetext{
${ }^{4}$ Though one may think of a simpler choice of the magnetic potential, [Ass20] explains the reason of defining it as in (2.3)-(2.5); this definition proves to be useful in explicitly deriving certain gauge results (see 5.1 and [Ass20, Proof of Lemma 4.3]).
} 
The potential $\mathbf{A}_{\alpha, a}$ is in $H^{1}\left(\mathbb{R}_{+}^{2} ; \mathbb{R}^{2}\right)$ and satisfies curl $\mathbf{A}_{\alpha, a}=\mathbb{1}_{D_{\alpha}^{1}}+a \mathbb{1}_{D_{\alpha}^{2}}$. The operator $\mathcal{H}_{\alpha, a}$ is defined over the domain

$$
\begin{aligned}
\operatorname{Dom} \mathcal{H}_{\alpha, a}=\left\{u \in L^{2}\left(\mathbb{R}_{+}^{2}\right):\right. & \left(\nabla-i \mathbf{A}_{\alpha, a}\right)^{j} u \in L^{2}\left(\mathbb{R}_{+}^{2}\right), \\
& \left.\quad \text { for } j \in\{1,2\},\left.\left(\nabla-i \mathbf{A}_{\alpha, a}\right) \cdot(0,1)\right|_{\partial\left(\mathbb{R}_{+}^{2}\right)}=0\right\} .
\end{aligned}
$$

Let $\mu(\alpha, a)$ be the bottom of the spectrum of $\mathcal{H}_{\alpha, a}$, defined by the min-max principle as follows:

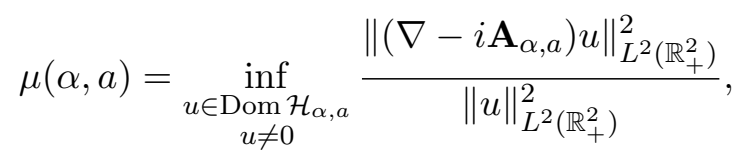

Remark 2.1. In [Ass20, Section 3], it is asserted that $\inf \operatorname{sp}_{e s s}\left(\mathcal{H}_{\alpha, a}\right)=|a| \Theta_{0}$. It follows that if $\mu(\alpha, a)<|a| \Theta_{0}$, then $\mu(\alpha, a)$ is an eigenvalue of $\mathcal{H}_{\alpha, a}$. This condition is crucial in deriving important properties of the effective energy in Section 3 below.

\section{EFFECTIVE ENERGY}

Let $a \in[-1,1) \backslash\{0\}, \alpha \in(0, \pi)$ and $b>0$. We introduce the following energy

$$
J_{b, \alpha, a}(u)=\int_{\mathbb{R}_{+}^{2}}\left(b\left|\left(\nabla-i \mathbf{A}_{\alpha, a}\right) u\right|^{2}-|u|^{2}+\frac{1}{2}|u|^{4}\right) d x,
$$

where $\mathbf{A}_{a, \alpha}$ is the vector potential in Section 2. This energy is defined over the space

$$
H_{\mathbf{A}_{\alpha, a}}^{1}=\left\{u \in L^{2}\left(\mathbb{R}_{+}^{2}\right):\left(\nabla-i \mathbf{A}_{\alpha, a}\right) u \in L^{2}\left(\mathbb{R}_{+}^{2}\right)\right\} .
$$

In what follows, we assume that $b>\left(|a| \Theta_{0}\right)^{-1}$. The functional in (3.1) is bounded from below. This can be seen by using the spectral properties of the Neumann realization of the Schrödinger operator $\left(\nabla-i \mathbf{A}_{\alpha, a}\right)^{2}$ on $\mathbb{R}_{+}^{2}$, involving those of other operators with uniform/step magnetic fields on $\mathbb{R}^{2}$ or $\mathbb{R}_{+}^{2}$ (see [Ass20, Section 2]). Indeed, consider a function $u \in C_{0}^{\infty}\left(\mathbb{R}^{2}\right)$ and let $\Gamma_{\alpha}:=\partial D_{\alpha}^{1} \cap \partial D_{\alpha}^{2}$.

- If $\operatorname{supp} u \Subset D_{\alpha}^{1} \cup D_{\alpha}^{2}$, then

$$
\int_{\mathbb{R}_{+}^{2}}\left|\left(\nabla-i \mathbf{A}_{\alpha, a}\right) u\right|^{2} d x \geq|a| \int_{\mathbb{R}_{+}^{2}}|u|^{2} d x>|a| \Theta_{0} \int_{\mathbb{R}_{+}^{2}}|u|^{2} d x
$$

having $\Theta_{0} \in(0,1)$.

- If $\operatorname{supp} u \Subset \mathbb{R}^{2} \backslash \Gamma_{\alpha}$ and meets the line $y=0$, then

$$
\int_{\mathbb{R}_{+}^{2}}\left|\left(\nabla-i \mathbf{A}_{\alpha, a}\right) u\right|^{2} d x \geq|a| \Theta_{0} \int_{\mathbb{R}_{+}^{2}}|u|^{2} d x
$$

- If $\operatorname{supp} u \Subset \mathbb{R}_{+}^{2}$ and meets $\Gamma_{\alpha}$, then

$$
\int_{\mathbb{R}_{+}^{2}}\left|\left(\nabla-i \mathbf{A}_{\alpha, a}\right) u\right|^{2} d x \geq \beta_{a} \int_{\mathbb{R}_{+}^{2}}|u|^{2} d x \geq|a| \Theta_{0} \int_{\mathbb{R}_{+}^{2}}|u|^{2} d x,
$$

where $\beta_{a}$ is the bottom of the spectrum of an operator, $\mathcal{L}_{a}$, with a step magnetic field defined over $\mathbb{R}^{2}$ in [Ass20, Section 2.2].

Consequently, the lower bound of $J_{b, \alpha, a}$ is derived as follows. Let $u \in H_{\mathbf{A}_{\alpha, a}}^{1}$ and $R>0$. One can find a partition of unity $\left(\chi_{k}\right)_{k \in \mathbb{N}}$ of $\mathbb{R}^{2}$, satisfying

$$
\sum_{k}\left|\chi_{k}\right|^{2}=1, \quad \sum_{k}\left|\nabla \chi_{k}\right|^{2} \leq C R^{-2}, \quad \text { and } \operatorname{supp} \chi_{k} \subset B_{k}(R),
$$


where $\left(B_{k}\right)_{k}$ are balls of radii $R$ such that $B_{1}(R)=B(O, R)$. Using the IMS localization formula (see [CFKS09, Theorem 3.2]), we have

$$
\begin{aligned}
J_{b, \alpha, a}(u) \geq & \sum_{k} J_{b, \alpha, a}\left(\chi_{k} u\right)-b \sum_{k}\left\|\nabla \chi_{k} u\right\|_{L^{2}\left(\mathbb{R}_{+}^{2}\right)}^{2} \\
\geq & \sum_{k \neq 1}\left(J_{b, \alpha, a}\left(\chi_{k} u\right)-\frac{C b}{R^{2}}\left\|\chi_{k} u\right\|_{L^{2}\left(\mathbb{R}_{+}^{2}\right)}^{2}\right)+J_{b, \alpha, a}\left(\chi_{1} u\right)-\frac{C b}{R^{2}}\left\|\chi_{1} u\right\|_{L^{2}\left(B_{1}(R)\right)}^{2} \\
\geq & \left(b|a| \Theta_{0}-1-\frac{C b}{R^{2}}\right) \sum_{k \neq 1}\left\|\chi_{k} u\right\|_{L^{2}\left(\mathbb{R}_{+}^{2}\right)}^{2}+\frac{1}{2}\left\|\left(\left|\chi_{1} u\right|^{2}-\left(1+\frac{C b}{R^{2}}\right)\right)\right\|_{L^{2}\left(B_{1}(R)\right)}^{2} \\
& -\frac{1}{2}\left(1+\frac{C b}{R^{2}}\right)^{2}\left|B_{1}(R)\right|, \quad(\text { by }(3.2)-(3.4)) \\
\geq & \left(b|a| \Theta_{0}-1-\frac{C b}{R^{2}}\right) \sum_{k \neq 1}\left\|\chi_{k} u\right\|_{L^{2}\left(\mathbb{R}_{+}^{2}\right)}^{2}-\frac{C R^{2}}{2}\left(1+\frac{C b}{R^{2}}\right)^{2} .
\end{aligned}
$$

Having $b>\left(|a| \Theta_{0}\right)^{-1}$, we choose $R$ sufficiently large so that $b|a| \Theta_{0}-1-C b / R^{2}>0$. Hence, we get the desired lower bound.

We define now the (finite) ground-state energy

$$
E_{\alpha, a}(b)=\inf _{u \in H_{\mathbf{A}_{\alpha, a}}^{1}} J_{b, \alpha, a}(u) .
$$

Working under the assumption

$$
\mu(\alpha, a)<|a| \Theta_{0},
$$

where $\mu(\alpha, a)$ is the value in (2.6), important properties of the functional in (3.1) are the following (cf. [FH10, Proposition 15.3.10] for the same argument). This functional is non-positive. It has non-trivial minimizers, $u_{b, \alpha, a}$, if and only if ${ }^{5}$

$$
\frac{1}{|a| \Theta_{0}}<b<\frac{1}{\mu(\alpha, a)} .
$$

These minimizers satisfy

$$
\left\|u_{b, \alpha, a}\right\|_{L^{\infty}\left(\mathbb{R}_{+}^{2}\right)} \leq 1
$$

and

$$
\int_{\mathbb{R}_{+}^{2}} e^{2 \delta|x|}\left(\left|u_{b, \alpha, a}(x)\right|^{2}+\left|\left(\nabla-i \mathbf{A}_{\alpha, a}\right) u_{b, \alpha, a}(x)\right|^{2}\right) d x \leq C,
$$

where $\delta, C$ are two positive constants dependent of $b, \alpha$ and $a$.

In light of the discussion above, we have

$$
\begin{aligned}
& E_{\alpha, a}(b)<0, \quad \text { for } \frac{1}{|a| \Theta_{0}}<b<\frac{1}{\mu(\alpha, a)}, \\
& E_{\alpha, a}(b)=0, \quad \text { for } b \geq \frac{1}{\mu(\alpha, a)} .
\end{aligned}
$$

Remark. Using a symmetry argument in the particular case where $\alpha=\pi / 2$ and $a=-1, E_{\pi / 2,-1}(b)$ will be the same effective energy introduced in [BNF07] (up to a scaling factor).

\footnotetext{
${ }^{5}$ Note that the condition in (3.7) is only valid under (3.6). In light of Remark 2.1, the eigenfunction corresponding to $\mu(\alpha, a)$ is used, in such situations, to construct a test function proving the non-triviality of the minimizers. If (3.6) is not satisfied, then $\mu(\alpha, a)=|a| \Theta_{0}$. In this case, for $b>\left(|a| \Theta_{0}\right)^{-1}, J_{b, \alpha, a}$ will only have zero minimizers, and the study will be trivial.
} 


\section{Preliminaries}

In this section, we present some properties of the minimizers $(\psi, \mathbf{A})$ of $\mathcal{E}_{\kappa, H}$ in (1.1), needed in the derivation our main results.

4.1. Classical Estimates. Recall the magnetic field $B_{0}$ introduced in Assumption 1.1. We fix a magnetic potential $\mathbf{F} \in H_{\text {div }}^{1}(\Omega)$ generating $B_{0}$ (see [AK16, Lemma A.1]):

$$
\exists \text { unique } \mathbf{F} \in H_{\mathrm{div}}^{1}(\Omega) \text { such that } \operatorname{curl} \mathbf{F}=B_{0} .
$$

The theorem below introduces some estimates of the critical points $(\psi, \mathbf{A})$ of $\mathcal{E}_{\kappa, H}$ in (1.1), involving the field $\mathbf{F}$. In light of this theorem, $\mathbf{F}$ proves to be a useful approximation of any vector potential $\mathbf{A}$ corresponding to a minimizer of (1.1).

Theorem 4.1. Let $\gamma \in(0,1)$ be a constant. Under Assumption 1.1, there exists a constant $C>0$ such that if $(\psi, \mathbf{A}) \in H^{1}(\Omega ; \mathbb{C}) \times H_{\text {div }}^{1}(\Omega)$ is a weak solution of (1.3), then

(1) $\|\psi\|_{L^{\infty}(\Omega)} \leq 1$,

(2) $\|(\nabla-i \kappa H \mathbf{A}) \psi\|_{L^{2}(\Omega)} \leq C \kappa\|\psi\|_{L^{2}(\Omega)}$,

(3) $\|\operatorname{curl}(\mathbf{A}-\mathbf{F})\|_{L^{2}(\Omega)} \leq \frac{C}{H}\|\psi\|_{L^{2}(\Omega)}^{2}$,

and, under the additional Assumption 1.3

(4) $\mathbf{A}-\mathbf{F} \in H^{2}(\Omega)$ and $\|\mathbf{A}-\mathbf{F}\|_{H^{2}(\Omega)} \leq \frac{C}{\kappa}$,

(5) $\mathbf{A}-\mathbf{F} \in C^{0, \gamma}(\bar{\Omega})$ and $\|\mathbf{A}-\mathbf{F}\|_{C^{0, \gamma}(\bar{\Omega})} \leq \frac{C}{\kappa}$.

The proof of this theorem can be found in [AK16, Theorem 4.2] and [FH10, Proposition 10.3.1 \& Lemma 10.3.2].

4.2. Exponential decay of the order parameter. The following theorem displays regimes for the intensity of the applied magnetic field where the order parameter and the GL energy are exponentially small away from certain intersection points of the magnetic edge and the boundary.

We suppose that the assumptions in Section 1.3 are satisfied. Let $j \in\{1, \cdots, n\}$. We define the set

$$
S=\left\{\mathrm{p}_{j} \in \Gamma \cap \partial \Omega: b<\left(\mu\left(\alpha_{j}, a\right)\right)^{-1}\right\} .
$$

Theorem 4.2. Assume that $b$ satisfy

$$
\left(|a| \Theta_{0}\right)^{-1}<b<\left(\min _{j \in\{1, \cdots, n\}} \mu\left(\alpha_{j}, a\right)\right)^{-1} .
$$

There exist constants $\kappa_{0}>0, C>0$, and $\delta_{0}>0$ such that, if

$$
\kappa \geq \kappa_{0}, \kappa_{0} \kappa^{-1} \leq \ell<1 \text {, and }(\psi, \mathbf{A}) \text { is a solution of (1.3), }
$$

then

$$
\int_{\Omega \cap\{\operatorname{dist}(x, S) \geq \ell\}}\left(|\psi|^{2}+(\kappa H)^{-1}|(\nabla-i \kappa H \mathbf{A}) \psi|^{2}\right) d x \leq C \kappa^{-1} e^{-\delta_{0} \kappa \ell} .
$$

Proof of Theorem 4.2. The proof is a consequence of the decay estimates established in [Ass20, Theorem 1.6]; indeed, for $b \in\left(\left(|a| \Theta_{0}\right)^{-1},\left(\min _{j \in\{1, \cdots, n\}} \mu\left(\alpha_{j}, a\right)\right)^{-1}\right)$, 
there exist $\kappa_{0}, C, \beta>0$ such that, for $\kappa \geq \kappa_{0}$ and $H=b \kappa$,

$$
\begin{aligned}
\int_{\Omega \cap\left\{\operatorname{dist}(x, S) \geq \frac{1}{\sqrt{\kappa H}}\right\}} e^{\beta \sqrt{\kappa H} \operatorname{dist}(x, S)}\left(|\psi|^{2}+\frac{1}{\kappa H}|(\nabla-i \kappa H \mathbf{A}) \psi|^{2}\right) d x & \\
& \leq C \int_{\Omega \cap\left\{\operatorname{dist}(x, S) \leq \frac{1}{\sqrt{\kappa H}}\right\} .}|\psi|^{2} d x .
\end{aligned}
$$

We choose $\kappa_{0}$ so that $\kappa_{0} \geq 1 / \sqrt{b}$. Thus, for $\kappa \geq \kappa_{0}$ and $\kappa_{0} \kappa^{-1} \leq \ell<1$, we get $\ell \geq 1 / \sqrt{\kappa H}$. Using (4.2), one can easily verify the claim of Theorem 4.2 , with $\delta_{0}=\delta_{0}(b)=\beta \sqrt{b}$.

\section{Local estimates of minimizers (Proof of Theorem 1.5)}

We are still working under the assumptions in Section 1.3. The aim of this section is to study the concentration of the minimizers $(\psi, \mathbf{A})$ of the functional (1.1) near the intersection points, $\mathbf{p}_{j}$, of $\partial \Omega$ and $\Gamma$. This will be displayed by local estimates of the GL ground-state energy and the $L^{4}$-norm of minimizers, that establish Theorem 1.5.

5.1. Change of variables. We will carry out the computation in adapted coordinates near $\partial \Omega \cap \Gamma$. The properties in this section are borrowed from [Ass20, Section 4], where these coordinates are first defined. We present them below for the convenience of the reader, and we refer to the aforementioned paper for more details.

For $j \in\{1, \cdots, n\}$, there exist $r_{j}>0$ and a local diffeomorphism $\Psi=\Psi_{j}$ of $\mathbb{R}^{2}$ satisfying the following:

$$
\Psi\left(\mathrm{p}_{j}\right)=(0,0), \quad\left|J_{\Psi}\right|\left(\mathrm{p}_{j}\right)=\left|J_{\Psi^{-1}}\right|(0,0)=1,
$$

and there exists a neighbourhood $\mathcal{U}_{j}$ of $(0,0)$ such that

$$
\begin{gathered}
\Psi\left(B\left(\mathrm{p}_{j}, r_{j}\right) \cap \Omega_{1}\right)=\mathcal{U}_{j} \cap D_{1}^{\alpha_{j}}, \quad \Psi\left(B\left(\mathrm{p}_{j}, r_{j}\right) \cap \Omega_{2}\right)=\mathcal{U}_{j} \cap D_{2}^{\alpha_{j}}, \\
\Psi\left(B\left(\mathrm{p}_{j}, r_{j}\right) \cap\left(\partial \Omega_{1} \backslash \Gamma\right)\right)=\mathcal{U}_{j} \cap \mathbb{R}_{+} \times\{0\}, \\
\Psi\left(B\left(\mathrm{p}_{j}, r_{j}\right) \cap\left(\partial \Omega_{2} \backslash \Gamma\right)\right)=\mathcal{U}_{j} \cap \mathbb{R}_{-} \times\{0\}, \\
\Psi\left(B\left(\mathrm{p}_{j}, r_{j}\right) \cap \Gamma\right)=\mathcal{U}_{j} \cap\left(\hat{x}_{2}=\hat{x}_{1} \tan \alpha_{j}\right) .
\end{gathered}
$$

Here, $\left(\hat{x}_{1}, \hat{x}_{2}\right):=\Psi\left(x_{1}, x_{2}\right)$, and the sets $D_{1}^{\alpha_{j}}$ and $D_{2}^{\alpha_{j}}$ are defined in (2.1). We assume further that the radii $r_{j}$ are sufficiently small so that $\left(B\left(\mathrm{p}_{j}, r_{j}\right)\right)_{j \in\{1, \cdots, n\}}$ is a family of disjoint balls. Using the properties above, one can prove the existence of a constant $C>0$, independent of $j$, such that the Jacobians $J_{\Psi}$ and $J_{\Psi^{-1}}$ satisfy

$$
|| J_{\Psi}(x)|-1| \leq C \ell \quad \text { and } \quad|| J_{\Psi^{-1}}(\hat{x})|-1| \leq C \ell,
$$

for all $x \in B\left(\mathrm{p}_{j}, \ell\right) \subset B\left(\mathrm{p}_{j}, r_{j}\right)$ and $\hat{x}=\Psi(x)$. Let $\mathbf{E}=\left(E_{1}, E_{2}\right) \in H^{1}\left(\Omega ; \mathbb{R}^{2}\right)$ be such that curl $\mathbf{E}=B$, for $B \in L^{2}\left(\mathbb{R}^{2}\right)$, and $u \in H^{1}(\Omega ; \mathbb{C})$ such that supp $u \subset B\left(\mathrm{p}_{j}, r_{j}\right)$. Consider the magnetic potential $\hat{\mathbf{E}}=\left(\hat{E}_{1}, \hat{E}_{2}\right) \in H^{1}\left(\Psi\left(B\left(\mathrm{p}_{j}, r_{j}\right)\right) \cap \mathbb{R}_{+}^{2} ; \mathbb{R}^{2}\right)$ satisfying $\hat{E}_{1} d \hat{x}_{1}+\hat{E}_{2} d \hat{x}_{2}=E_{1} d x_{1}+E_{2} d x_{2}$, and the function $\hat{u}$, defined in $\Psi\left(B\left(\mathrm{p}_{j}, r_{j}\right)\right) \cap$ $\mathbb{R}_{+}^{2}$ by $\hat{u}(\hat{x})=u\left(\Psi^{-1}(\hat{x})\right)$. Furthermore, let

$$
\hat{B}(\hat{x})=B\left(\Psi^{-1}(\hat{x})\right), \quad \text { for all } \hat{x} \in \Psi\left(B\left(\mathrm{p}_{j}, r_{j}\right)\right) \cap \mathbb{R}_{+}^{2} .
$$

One can check that

$$
\operatorname{curl} \hat{\mathbf{E}}=\partial_{\hat{x}_{1}} \hat{E}_{2}-\partial_{\hat{x}_{2}} \hat{E}_{1}=\hat{B} J_{\Psi^{-1}}
$$


and, for any $\mathfrak{b}>0$

$$
\begin{aligned}
& \int_{\Omega}|(\nabla-i \mathfrak{b} \mathbf{E}) u|^{2} d x= \\
& \quad \int_{D} \sum_{1 \leq k, m \leq 2} G_{k, m}(\hat{x})\left(\partial_{\hat{x}_{k}}-i \mathfrak{b} \hat{E}_{k}\right) \hat{u}(\hat{x}) \overline{\left(\partial_{\hat{x}_{m}}-i \mathfrak{b} \hat{E}_{m}\right) \hat{u}(\hat{x})}\left|J_{\Psi^{-1}}(\hat{x})\right| d \hat{x} .
\end{aligned}
$$

Here $D=\Psi\left(B\left(\mathrm{p}_{j}, r_{j}\right)\right) \cap \mathbb{R}_{+}^{2}$ and $G_{k, m}(\hat{x})$ are the elements of the matrix $G(\hat{x})=$ $\left.(d \Psi)(d \Psi)^{t}\right|^{-1}(\hat{x})$. For any $\ell<r_{j}$, we have

$$
\left|G_{k, m}(\hat{x})-\delta_{k, m}\right| \leq C \ell, \quad \hat{x} \in \Psi\left(B\left(\mathrm{p}_{j}, \ell\right)\right)
$$

for some $C>0$ independent of $j$.

Using the coordinates transformation above, the field $\mathbf{F}$ in (4.1) can be expressed in the following canonical manner:

Lemma 5.1. Let $a \in[-1,1) \backslash\{0\}$, and $B(0, l) \subset \Psi\left(B\left(\mathrm{p}_{j}, r_{j}\right)\right)$ be a ball of radius l. Consider the vector potential $\mathbf{F} \in H_{\text {div }}^{1}(\Omega)$ satisfying curl $\mathbf{F}=\mathbb{1}_{\Omega_{1}}+a \mathbb{1}_{\Omega_{2}}$. There exists a function $\varphi_{j, l} \in H^{2}\left(B(0, l) \cap \mathbb{R}_{+}^{2}\right)$ such that the vector potential $\hat{\mathbf{F}}_{\mathrm{g}}:=\hat{\mathbf{F}}$ $\nabla_{\hat{x}_{1}, \hat{x}_{2}} \varphi_{j, l}$, defined in $B(0, l) \cap \mathbb{R}_{+}^{2}$, satisfies

$$
\left(\hat{F}_{\mathrm{g}}\right)_{1}=0, \quad\left(\hat{F}_{\mathrm{g}}\right)_{2}=A_{\alpha, a}+f
$$

where $A_{\alpha, a}$ is the potential introduced in (2.2), $f$ is a continuous function satisfying $\left|f\left(\hat{x}_{1}, \hat{x}_{2}\right)\right| \leq C\left(\hat{x}_{1}^{2}+\left|\hat{x}_{1} \hat{x}_{2}\right|\right)$, for some $C>0$ independent of $j$.

5.2. A useful lower bound. We introduce the following local energies of any configuration $(\psi, \mathbf{A}) \in H^{1}(\Omega ; \mathbb{C}) \times H_{\text {div }}^{1}(\Omega)$, in a domain $D \subset \Omega$

$$
\begin{aligned}
\mathcal{E}_{0}(\psi, \mathbf{A} ; D) & =\int_{D}\left(|(\nabla-i \kappa H \mathbf{A}) \psi|^{2}-\kappa^{2}|\psi|^{2}+\frac{1}{2} \kappa^{2}|\psi|^{4}\right) d x, \\
\mathcal{E}(\psi, \mathbf{A} ; D) & =\mathcal{E}_{0}(\psi, \mathbf{A} ; D)+(\kappa H)^{2} \int_{\Omega}|\operatorname{curl}(\mathbf{A}-\mathbf{F})|^{2} d x .
\end{aligned}
$$

Let $\ell \in(0,1)$. For each $\mathrm{p}_{j} \in \Gamma \cap \partial \Omega, j \in\{1, \cdots, n\}$, recall the set $\mathcal{N}_{j}(\ell)$ defined in (1.5) by

$$
\mathcal{N}_{j}(\ell)=\left\{x \in \Omega, \operatorname{dist}\left(x, \mathrm{p}_{j}\right) \leq \ell\right\} .
$$

In Proposition 5.2 below, we establish a lower bound for the local energy of an arbitrary function $u \in H^{1}(\Omega ; \mathbb{C})$ supported in a neighbourhood of $\mathrm{p}_{j}$, which will be helpful in deriving the local estimates in Theorem 1.5.

Proposition 5.2. There exist two constants $\kappa_{0}>1$ and $C>0$ such that, for $\kappa \geq \kappa_{0}$ and for any $\mathrm{p}_{j} \in \Gamma \cap \partial \Omega$, the following is true. If

- $(\psi, \mathbf{A}) \in H^{1}(\Omega ; \mathbb{C}) \times H_{\text {div }}^{1}(\Omega)$ is a solution of $(1.3)$.

- $u \in H^{1}(\Omega ; \mathbb{C})$ such that $\operatorname{supp} u \subset B\left(\mathrm{p}_{j}, \ell\right)$ and $|u| \leq 1$.

then

$$
\mathcal{E}_{0}\left(u, \mathbf{A} ; \mathcal{N}_{j}(\ell)\right) \geq b^{-1} E_{\alpha_{j}, a}(b)-C\left(\kappa^{\frac{3}{2}} \ell^{2}+\kappa^{\frac{5}{2}} \ell^{\frac{10}{3}}+\kappa^{2} \ell^{3}+\kappa^{\frac{9}{2}} \ell^{6}\right),
$$

where $\mathcal{E}_{0}$ is the functional in (5.4), and $E_{\alpha_{j}, a}(b)$ is the energy in (3.5).

Proof. Let $\gamma \in(0,1)$ and $\mathbf{F}$ be the vector field introduced in (4.1). We define the function $\phi_{j}$ by

$$
\phi_{j}(x)=\left(\mathbf{A}\left(\mathbf{p}_{j}\right)-\mathbf{F}\left(\mathrm{p}_{j}\right)\right) \cdot x .
$$

As a consequence of the fifth item in Theorem 4.1, we get the following approximation of the vector potential $\mathbf{A}$

$$
\left|\mathbf{A}(x)-\nabla \phi_{j}(x)-\mathbf{F}(x)\right| \leq \frac{C}{\kappa} \ell^{\gamma}, \quad \text { for } x \in \mathcal{N}_{j}(\ell) .
$$


We choose $\gamma=2 / 3$ in (5.7). Let $v=e^{-i \kappa H \phi_{j}} u$. Using (5.7), Cauchy's inequality, and the bound $|v| \leq 1$, we may write

$$
\mathcal{E}_{0}\left(u, \mathbf{A} ; \mathcal{N}_{j}(\ell)\right) \geq\left(1-\kappa^{-\frac{1}{2}}\right) \mathcal{E}_{0}\left(v, \mathbf{F} ; \mathcal{N}_{j}(\ell)\right)-C\left(\kappa^{\frac{3}{2}} \ell^{2}+\kappa^{\frac{5}{2}} \ell^{\frac{10}{3}}\right) .
$$

Now, we use the change of variables introduced in Section 5.1, valid in a neighbourhood of $\mathrm{p}_{j}$, to locally send the domain in $\Omega$ onto $\mathbb{R}_{+}^{2} . \kappa$ is assumed sufficiently large so that $B\left(\mathrm{p}_{j}, \ell\right) \subset B\left(\mathrm{p}_{j}, r_{j}\right)$. We associate to $v$ the function $\hat{v}=v \circ \Psi^{-1}$, defined in $\Psi\left(B\left(\mathrm{p}_{j}, \ell\right)\right)$. We may use the transformation formula in (5.2) and the properties in (5.1) and (5.3) to conclude that

$$
\begin{aligned}
(1-C \ell) \int_{\Psi\left(B\left(\mathrm{p}_{j}, \ell\right)\right) \cap \mathbb{R}_{+}^{2}}|(\nabla-i \kappa H \hat{\mathbf{F}}) \hat{v}|^{2} d \hat{x} \leq \int_{\Omega}|(\nabla-i \kappa H \mathbf{F}) v|^{2} d x \\
\leq(1+C \ell) \int_{\Psi\left(B\left(\mathrm{p}_{j}, \ell\right)\right) \cap \mathbb{R}_{+}^{2}}|(\nabla-i \kappa H \hat{\mathbf{F}}) \hat{v}|^{2} d \hat{x}
\end{aligned}
$$

where $\hat{\mathbf{F}}$ is the transform of $\mathbf{F}$ by $\Psi$, and $C>0$ is a constant independent of $j$. In addition, due to the support of $v$ and (5.1), we note the existence of $c_{1}>0$ such that $\Psi\left(B\left(\mathrm{p}_{j}, \ell\right)\right) \subset B\left(0, c_{1} \ell\right) \subset \Psi\left(B\left(\mathrm{p}_{j}, r_{j}\right)\right)$, for large $\kappa$. Consequently, the gauge transform in Lemma 5.1 allows us to write

$$
\begin{aligned}
\int_{\Psi\left(B\left(\mathbf{p}_{j}, \ell\right)\right) \cap \mathbb{R}_{+}^{2}}|(\nabla-i \kappa H \hat{\mathbf{F}}) \hat{v}|^{2} d \hat{x} & \\
& =\int_{\Psi\left(B\left(\mathrm{p}_{j}, \ell\right)\right) \cap \mathbb{R}_{+}^{2}}\left|\left(\nabla-i \kappa H \hat{\mathbf{F}}_{\mathrm{g}}\right) \hat{v}_{\mathrm{g}}\right|^{2} d \hat{x},
\end{aligned}
$$

where $\hat{v}_{\mathrm{g}}(\hat{x})=\hat{v}(\hat{x}) e^{-i \kappa H \varphi(\hat{x})}$, for $\hat{x} \in \Psi\left(B\left(\mathrm{p}_{j}, \ell\right)\right) \cap \mathbb{R}_{+}^{2}$. Here $\varphi=\varphi_{j, l}$, for $l=$ $c_{1} \ell$, is the gauge function in Lemma 5.1 , and $\hat{\mathbf{F}}_{\mathrm{g}}$ is the magnetic potential in the aforementioned lemma.

Recall the potential $\mathbf{A}_{\alpha, a}$ introduced in (2.2). Extending $\hat{v}$ and $\hat{v}_{\mathrm{g}}$ by zero in $\mathbb{R}_{+}^{2}$, the Cauchy's inequality applied in (5.10), and the support of the function $\hat{v}_{\mathrm{g}}$ imply

$$
\begin{array}{r}
\int_{\Psi\left(B\left(\mathbf{p}_{j}, \ell\right)\right) \cap \mathbb{R}_{+}^{2}}\left|\left(\nabla-i \kappa H \hat{\mathbf{F}}_{\mathrm{g}}\right) \hat{v}_{\mathrm{g}}\right|^{2} d \hat{x} \geq\left(1-\kappa^{-\frac{1}{2}}\right) \int_{\mathbb{R}_{+}^{2}}\left|\left(\nabla-i \kappa H \mathbf{A}_{\alpha_{j}, a}\right) \hat{v}_{\mathrm{g}}\right|^{2} d \hat{x} \\
-C \kappa^{\frac{9}{2}} \ell^{4} \int_{\mathbb{R}_{+}^{2}}\left|\hat{v}_{\mathrm{g}}\right|^{2} d \hat{x}, \quad \text { (5 }
\end{array}
$$

where $\alpha_{j}$ is the corresponding angle to the point $\mathrm{p}_{j}$, defined in Notation 1.2. But

$$
\int_{\mathbb{R}_{+}^{2}}\left|\hat{v}_{\mathrm{g}}\right|^{2} d \hat{x}=\int_{B\left(\mathbf{p}_{j}, \ell\right) \cap \Omega}|v|^{2}\left|J_{\Psi}\right| d x .
$$

Thus, using (5.1) we get

$$
(1-C \ell) \int_{\Omega}|v|^{2} d x \leq \int_{\mathbb{R}_{+}^{2}}\left|\hat{v}_{\mathrm{g}}\right|^{2} d \hat{x} \leq(1+C \ell) \int_{\Omega}|v|^{2} d x .
$$

Plug (5.12) into (5.11), and use again $|v| \leq 1$ together with its support to obtain

$$
\int_{\Psi\left(B\left(\mathbf{p}_{j}, \ell\right)\right) \cap \mathbb{R}_{+}^{2}}\left|\left(\nabla-i \kappa H \hat{\mathbf{F}}_{\mathrm{g}}\right) \hat{v}_{\mathrm{g}}\right|^{2} d \hat{x} \geq\left(1-\kappa^{-\frac{1}{2}}\right) \int_{\mathbb{R}_{+}^{2}}\left|\left(\nabla-i \kappa H \mathbf{A}_{\alpha_{j}, a}\right) \hat{v}_{\mathrm{g}}\right|^{2} d \hat{x}-C \kappa^{\frac{9}{2}} \ell^{6} .
$$

Similarly to (5.12), we have

$$
(1-C \ell) \int_{\Omega}|v|^{4} d x \leq \int_{\mathbb{R}_{+}^{2}}\left|\hat{v}_{g}\right|^{4} d \hat{x} \leq(1+C \ell) \int_{\Omega}|v|^{4} d x .
$$


Consequently, using (5.9), (5.10) and (5.12)-(5.14), we retake the energy $\mathcal{E}_{0}\left(v, \mathbf{F} ; \mathcal{N}_{j}(\ell)\right)$ appearing in (5.8) and write

$$
\begin{aligned}
& \mathcal{E}_{0}\left(v, \mathbf{F} ; \mathcal{N}_{j}(\ell)\right)=\int_{\Omega}\left(|(\nabla-i \kappa H \mathbf{F}) v|^{2}-\kappa^{2}|v|^{2}+\frac{\kappa^{2}}{2}|v|^{4}\right) d x \\
& \geq\left(1-C \ell-\kappa^{-\frac{1}{2}}\right)\left(\int_{\mathbb{R}_{+}^{2}}\left(\left|\left(\nabla-i \kappa H \mathbf{A}_{\alpha_{j}, a}\right) \hat{v}_{\mathrm{g}}\right|^{2}-\kappa^{2}\left|\hat{v}_{\mathrm{g}}\right|^{2}+\frac{\kappa^{2}}{2}\left|\hat{v}_{\mathrm{g}}\right|^{4}\right) d \hat{x}\right)-r(\kappa),
\end{aligned}
$$

where $r(\kappa)=C\left(\kappa^{\frac{3}{2}} \ell^{2}+\kappa^{\frac{9}{2}} \ell^{6}+\kappa^{2} \ell^{3}\right)$. Next, we use the scaling $t=\sqrt{\kappa H} \hat{x}=\sqrt{b} \kappa \hat{x}$ (see Assumption 1.3), and define

$$
\mathrm{v}(t)=\hat{v}_{\mathrm{g}}\left(\frac{t}{\sqrt{b} \kappa}\right), \quad \text { for } t \in \mathbb{R}_{+}^{2} .
$$

One can simply check that

$$
\int_{\mathbb{R}_{+}^{2}}\left|\left(\nabla_{\hat{x}}-i \kappa H \mathbf{A}_{\alpha_{j}, a}(\hat{x})\right) \hat{v}_{\mathrm{g}}(\hat{x})\right|^{2} d \hat{x}=\int_{\mathbb{R}_{+}^{2}}\left|\left(\nabla_{t}-i \mathbf{A}_{\alpha_{j}, a}(t)\right) \mathrm{v}(t)\right|^{2} d t
$$

and

$$
\int_{\mathbb{R}_{+}^{2}}\left|\hat{v}_{\mathrm{g}}(\hat{x})\right|^{2} d \hat{x}=\frac{1}{b \kappa^{2}} \int_{\mathbb{R}_{+}^{2}}|\mathrm{v}(t)|^{2} d t, \quad \int_{\mathbb{R}_{+}^{2}}\left|\hat{v}_{\mathrm{g}}(\hat{x})\right|^{4} d \hat{x}=\frac{1}{b \kappa^{2}} \int_{\mathbb{R}_{+}^{2}}|\mathrm{v}(t)|^{4} d t .
$$

Hence,

$$
\int_{\mathbb{R}_{+}^{2}}\left(\left|\left(\nabla-i \kappa H \mathbf{A}_{\alpha_{j}, a}\right) \hat{v}_{\mathrm{g}}\right|^{2}-\kappa^{2}\left|\hat{v}_{\mathrm{g}}\right|^{2}+\frac{\kappa^{2}}{2}\left|\hat{v}_{\mathrm{g}}\right|^{4}\right) d \hat{x}=\frac{1}{b} J_{b, \alpha_{j}, a}(\mathrm{v}) \geq \frac{1}{b} E_{\alpha_{j}, a}(b),
$$

where $J_{b, \alpha_{j}, a}$ and $E_{\alpha_{j}, a}(b)$ are the energies in (3.1) and (3.5) respectively. Having $E_{\alpha_{j}, a}(b) \leq 0$, we put (5.16) in (5.15) and get

$$
\mathcal{E}_{0}\left(v, \mathbf{F} ; \mathcal{N}_{j}(\ell)\right) \geq \frac{1}{b} E_{\alpha_{j}, a}(b)-C \kappa^{\frac{3}{2}} \ell^{2}-C \kappa^{\frac{9}{2}} \ell^{6}-C \kappa^{2} \ell^{3} .
$$

Implement (5.17) in (5.8) to complete the proof.

\subsection{Proof of the main result.}

Proof of Theorem 1.5. Let $\gamma \in(0,1)$ and $\hat{\ell}=(1+\gamma) \ell$. We assume that $\kappa$ is sufficiently large so that $\mathcal{N}_{j}(\hat{\ell}) \cap \mathcal{N}_{k}(\hat{\ell})=\emptyset$, for any $j, k \in\{1, \cdots, n\}, j \neq k$.

Let $j \in T$. Consider a smooth function $f_{j}$ satisfying

$$
f_{j}=1 \text { in } \mathcal{N}_{j}(\ell), f_{j}=0 \text { in } \mathcal{N}_{j}(\hat{\ell})^{\complement}, 0 \leq f_{j} \leq 1 \text { and }\left|\nabla f_{j}\right| \leq C \gamma^{-1} \ell^{-1} \text { in } \Omega \text {. }
$$

Here, $\mathcal{N}_{j}(\hat{\ell})^{\complement}$ denotes the complement of $\mathcal{N}_{j}(\hat{\ell})$ in $\Omega$. We have the following identity

$$
\begin{aligned}
\int_{\mathcal{N}_{j}(\hat{\ell})}\left|(\nabla-i \kappa H \mathbf{A}) f_{j} \psi\right|^{2} d x= & \int_{\mathcal{N}_{j}(\hat{\ell})} \mid \\
& \left.f_{j}(\nabla-i \kappa H \mathbf{A}) \psi\right|^{2} d x+\left.\int_{\mathcal{N}_{j}(\hat{\ell})}\left|\nabla f_{j}\right|^{2} \psi\right|^{2} d x \\
& +2 \operatorname{Re} \int_{\mathcal{N}_{j}(\hat{\ell})} f_{j}(\nabla-i \kappa H \mathbf{A}) \psi \cdot \psi \nabla f_{j} d x
\end{aligned}
$$

Consider the following obvious decompositions

$$
\begin{aligned}
\int_{\mathcal{N}_{j}(\hat{\ell})} f_{j}^{2}|\psi|^{2} d x & =\int_{\mathcal{N}_{j}(\hat{\ell})}|\psi|^{2} d x+\int_{\mathcal{N}_{j}(\hat{\ell})}\left(f_{j}^{2}-1\right)|\psi|^{2} d x \\
\int_{\mathcal{N}_{j}(\hat{\ell})} f_{j}^{4}|\psi|^{4} d x & =\int_{\mathcal{N}_{j}(\hat{\ell})}|\psi|^{4} d x+\int_{\mathcal{N}_{j}(\hat{\ell})}\left(f_{j}^{4}-1\right)|\psi|^{4} d x,
\end{aligned}
$$


and that of $\int_{\mathcal{N}_{j}(\hat{\ell})}\left|f_{j}(\nabla-i \kappa H \mathbf{A}) \psi\right|^{2} d x$ into

$$
\int_{\mathcal{N}_{j}(\hat{\ell})}|(\nabla-i \kappa H \mathbf{A}) \psi|^{2} d x+\int_{\mathcal{N}_{j}(\hat{\ell})}\left(f_{j}^{2}-1\right)|(\nabla-i \kappa H \mathbf{A}) \psi|^{2} d x .
$$

Moreover, note that

$$
\left|\operatorname{Re} \int_{\mathcal{N}_{j}(\hat{\ell})} f_{j}(\nabla-i \kappa H \mathbf{A}) \psi \cdot \psi \nabla f_{j} d x\right| \leq\left\|f_{j}(\nabla-i \kappa H \mathbf{A}) \psi\right\|_{L^{2}\left(\mathcal{N}_{j}(\hat{\ell})\right)}\left\|\psi \nabla f_{j}\right\|_{L^{2}\left(\mathcal{N}_{j}(\hat{\ell})\right)}
$$

and recall that $\ell \approx \kappa^{-\rho}$ is chosen so that $\ell \gg \kappa^{-1}$. Hence, using (5.19)-(5.21) and the properties of $f_{j}$ in (5.18), particularly that $\nabla f_{j}, f_{j}^{2}-1$ and $f_{j}^{4}-1$ are supported in $\mathcal{N}_{j}(\ell)^{\complement}$, together with Theorem 4.2 which ensures that $|\psi|$ is exponentially small in $\mathcal{N}_{j}(\hat{\ell}) \backslash \mathcal{N}_{j}(\ell)$, we get

$$
\mathcal{E}_{0}\left(f_{j} \psi, \mathbf{A} ; \mathcal{N}_{j}(\hat{\ell})\right)=\mathcal{E}_{0}\left(\psi, \mathbf{A} ; \mathcal{N}_{j}(\hat{\ell})\right)+o(1) .
$$

$A$ lower bound of the local energy. Notice that the function $f_{j} \psi$ satisfies the conditions in Proposition 5.2, with $\hat{\ell}$ replacing $\ell$, then using this proposition and (5.22), we get (with the choice of $\rho \in(4 / 5,1)$ in $\ell \approx \kappa^{-\rho}$ )

$$
\mathcal{E}_{0}\left(\psi, \mathbf{A} ; \mathcal{N}_{j}(\hat{\ell})\right) \geq \frac{1}{b} E_{\alpha_{j}, a}(b)+o(1) .
$$

An upper bound of the local energy. Inspired by [SS03, HK17, HK18, AKPS19], we define the following test function

$$
w(x)=\mathbb{1}_{\mathcal{N}_{j}(\hat{\ell})}(x) f_{j}(x) e^{i \kappa H\left(\phi_{j}(x)+\varphi_{j, \hat{\ell}}\left(\Psi_{j}(x)\right)\right.} u_{j}\left(\sqrt{\kappa H} \Psi_{j}(x)\right)+\left(1-f_{j}(x)\right) \psi(x),
$$

where $\Psi_{j}$ is the coordinate transformation in Section $5.1, f_{j}, \phi_{j}$, and $\varphi_{j, \hat{\ell}}$ are respectively the functions in (5.18), (5.6), and Lemma 5.1, and $u_{j}=u_{b, \alpha_{j}, a}$ is a minimizer of the functional in (3.1) with $b=H / \kappa$.

A minimizer $(\psi, \mathbf{A})$ of (1.1) obviously satisfies

$$
\mathcal{E}_{\kappa, H}(\psi, \mathbf{A}) \leq \mathcal{E}_{\kappa, H}(w, \mathbf{A}) .
$$

Suppressing the term $\kappa^{2} H^{2}\|\operatorname{curl}(\mathbf{A}-\mathbf{F})\|_{L^{2}(\Omega)}^{2}$ from the above expression, we get

$$
\mathcal{E}_{0}(\psi, \mathbf{A} ; \Omega) \leq \mathcal{E}_{0}(w, \mathbf{A} ; \Omega) .
$$

Note that $f_{j}=0$ in $\mathcal{N}_{j}(\hat{\ell})^{\complement}$, hence using the following decompositions

$$
\begin{aligned}
& \mathcal{E}_{0}(\psi, \mathbf{A} ; \Omega)=\mathcal{E}_{0}\left(\psi, \mathbf{A} ; \mathcal{N}_{j}(\hat{\ell})\right)+\mathcal{E}_{0}\left(\psi, \mathbf{A} ; \mathcal{N}_{j}(\hat{\ell})^{\complement}\right) \\
& \mathcal{E}_{0}(w, \mathbf{A} ; \Omega)=\mathcal{E}_{0}\left(w, \mathbf{A} ; \mathcal{N}_{j}(\hat{\ell})\right)+\mathcal{E}_{0}\left(\psi, \mathbf{A} ; \mathcal{N}_{j}(\hat{\ell})^{\complement}\right)
\end{aligned}
$$

we further get

$$
\mathcal{E}_{0}\left(\psi, \mathbf{A} ; \mathcal{N}_{j}(\hat{\ell})\right) \leq \mathcal{E}_{0}\left(w, \mathbf{A} ; \mathcal{N}_{j}(\hat{\ell})\right)
$$

On the other hand, the decay estimates in (3.8) and Theorem 4.2 assure that $u_{j}\left(\sqrt{\kappa H} \Psi_{j}(x)\right)$ and $|\psi(x)|$ are exponentially small in $\mathcal{N}_{j}(\hat{\ell}) \backslash \mathcal{N}_{j}(\ell)$ (having $\ell \gg \kappa^{-1}$ ). Hence, a computation ${ }^{6}$ quite similar to the one done in the lower bound proof above and in Proposition 5.2 yields

$$
\mathcal{E}_{0}\left(w, \mathbf{A} ; \mathcal{N}_{j}(\hat{\ell})\right)=\frac{1}{b} E_{\alpha_{j}, a}(b)+o(1) .
$$

\footnotetext{
${ }^{6}$ we omit the computation details which is now straightforward, after having the proofs in the above paragraph and Proposition 5.2.
} 
Put (5.25) in (5.24) to get the following upper bound

$$
\mathcal{E}_{0}\left(\psi, \mathbf{A} ; \mathcal{N}_{j}(\hat{\ell})\right) \leq \frac{1}{b} E_{\alpha_{j}, a}(b)+o(1) .
$$

The order parameter estimates. Integrating by parts in the first equation of (1.3), we get (see [FK11, (6.2)])

$$
\int_{\mathcal{N}_{j}(\hat{\ell})}\left(\left|(\nabla-i \kappa H \mathbf{A}) f_{j} \psi\right|^{2}-\left|\nabla f_{j}\right|^{2}|\psi|^{2}\right) d x=\kappa^{2} \int_{\mathcal{N}_{j}(\hat{\ell})}\left(|\psi|^{2}-|\psi|^{4}\right) f_{j}^{2} d x
$$

Consequently,

$$
\begin{aligned}
\mathcal{E}_{0}\left(f_{j} \psi, \mathbf{A} ; \mathcal{N}_{j}(\hat{\ell})\right)= & \kappa^{2} \int_{\mathcal{N}_{j}(\hat{\ell})} f_{j}^{2}\left(-1+\frac{1}{2} f_{j}^{2}\right)|\psi|^{4} d x+\int_{\mathcal{N}_{j}(\hat{\ell})}\left|\nabla f_{j}\right|^{2}|\psi|^{2} d x \\
= & -\frac{1}{2} \kappa^{2} \int_{\mathcal{N}_{j}(\ell)}|\psi|^{4} d x+\kappa^{2} \int_{\mathcal{N}_{j}(\hat{\ell}) \backslash \mathcal{N}_{j}(\ell)} f_{j}^{2}\left(-1+\frac{1}{2} f_{j}^{2}\right)|\psi|^{4} d x \\
& +\int_{\mathcal{N}_{j}(\hat{\ell})}\left|\nabla f_{j}\right|^{2}|\psi|^{2} d x .
\end{aligned}
$$

Again, using the exponential decay of $\psi$ in $\mathcal{N}_{j}(\hat{\ell}) \backslash \mathcal{N}_{j}(\ell)$, and the properties of $f_{j}$ in (5.18), we get

$$
\mathcal{E}_{0}\left(f_{j} \psi, \mathbf{A} ; \mathcal{N}_{j}(\hat{\ell})\right)=-\frac{1}{2} \kappa^{2} \int_{\mathcal{N}_{j}(\ell)}|\psi|^{4} d x+o(1) .
$$

Implement this equation in (5.22) and use the bounds in (5.23) and (5.26) to get the estimates of the $L^{4}$-norm of $\psi$ in (1.6).

The global energy estimates. Now, the estimates of the ground-state energy in (1.7) are easy to establish. First notice that

$$
\begin{aligned}
\mathrm{E}_{\text {g.st }}(\kappa, H) & =\mathcal{E}_{0}(\psi, \mathbf{A} ; \Omega)+\kappa^{2} H^{2}\|\operatorname{curl}(\mathbf{A}-\mathbf{F})\|_{L^{2}(\Omega)}^{2} \\
& =\mathcal{E}_{0}\left(\psi, \mathbf{A} ; \mathcal{S}_{\hat{\ell}}\right)+\kappa^{2} H^{2}\|\operatorname{curl}(\mathbf{A}-\mathbf{F})\|_{L^{2}(\Omega)}^{2}+o(1), \\
& =\frac{1}{b} \sum_{j \in T} E_{\alpha_{j}, a}(b)+\kappa^{2} H^{2}\|\operatorname{curl}(\mathbf{A}-\mathbf{F})\|_{L^{2}(\Omega)}^{2}+o(1),
\end{aligned}
$$

where $\mathcal{S}_{\hat{\ell}}:=\bigcup_{j \in T} \mathcal{N}_{j}(\hat{\ell})$. The equality above is obtained by summing over $T$ in (5.23) and (5.26), and due to the aforementioned decay of the minimizer and its energy in $\mathcal{S}_{\hat{\ell}}^{\complement}$. Moreover, this decay and Item 3 in Theorem 4.1 assure that

$$
\kappa^{2} H^{2}\|\operatorname{curl}(\mathbf{A}-\mathbf{F})\|_{L^{2}(\Omega)}^{2} \leq C \kappa^{2}\|\psi\|_{L^{2}(\Omega)}^{4} \leq \tilde{C} \kappa^{2} \ell^{4}=o(1) .
$$

This completes the proof of (1.7).

\section{ACKNOWLEDGEMENTS}

I would like to thank Ayman Kachmar for his valuable comments about this article.

\section{REFERENCES}

[AK16] W. Assaad and A. Kachmar. The influence of magnetic steps on bulk superconductivity. Discrete Contin. Dyn. Syst. Ser. A, 36:6623-6643, 2016.

[AKPS19] W. Assaad, A. Kachmar, and M. Persson-Sundqvist. The distribution of superconductivity near a magnetic barrier. Comm. Math. Phys., 366(1):269-332, 2019.

[AKS20] W. Assaad, A. Kashmar, and L. Sabbagh. Non-homogeneous magnetic permeability and magnetic steps within the Ginzburg-Landau model (submitted). 2020.

[Ass20] W. Assaad. The breakdown of superconductivity in the presence of magnetic steps. Commun. Contemp. Math., https://doi.org/10.1142/S0219199720500054, 2020.

[Att15a] K. Attar. Energy and vorticity of the Ginzburg-Landau model with variable magnetic field. Asymptot. Anal., 93(1-2):75-114, 2015. 
[Att15b] K. Attar. The ground state energy of the two dimensional Ginzburg-Landau functional with variable magnetic field. Ann. Inst. H. Poincaré Anal. Non Linéaire, 32(2):325-345, 2015.

[BND06] V. Bonnaillie-Noël and M. Dauge. Asymptotics for the low-lying eigenstates of the Schrödinger operator with magnetic field near corners. Ann. Henri Poincaré, 7(5):899931, 2006.

[BNF07] V. Bonnaillie-Noël and S. Fournais. Superconductivity in domains with corners. Rev. Math. Phys., 19(06):607-637, 2007.

[Bon05] V. Bonnaillie. On the fundamental state energy for a Schrödinger operator with magnetic field in domains with corners. Asymptot. Anal., 41(3-4):215-258, 2005.

[CDR17] M. Correggi, B. Devanarayanan, and N. Rougerie. Universal and shape dependent features of surface superconductivity. Eur. Phys. J. B, 90(11):231, 2017.

[CFKS09] H.L. Cycon, R.G. Froese, W. Kirsch, and B. Simon. Schrödinger Operators: With Application to Quantum Mechanics and Global Geometry. Springer, 2009.

[CG17] M. Correggi and E. L. Giacomelli. Surface superconductivity in presence of corners. Rev. Math. Phys., 29(2):1750005, 2017.

[CG19] M. Correggi and E.L. Giacomelli. Effects of corners in surface superconductivity. arXiv:1908.10112, 2019.

[CR14] M. Correggi and N. Rougerie. On the Ginzburg-Landau functional in the surface superconductivity regime. Commun. Math. Phys., 332(3):1297-1343, 2014.

[CR16a] M. Correggi and N. Rougerie. Boundary behavior of the Ginzburg-Landau order parameter in the surface superconductivity regime. Arch. Ration. Mech. Anal., 219(1):553-606, 2016.

[CR16b] M. Correggi and N. Rougerie. Effects of boundary curvature on surface superconductivity. Lett. Math. Phys., 106(4):445-467, 2016.

[DMR18] M. Dauge, J.P. Miqueu, and N. Raymond. On the semiclassical laplacian with magnetic field having self-intersecting zero set. arXiv:1807.09028, 2018.

[Erd97] László Erdôs. Dia-and paramagnetism for nonhomogeneous magnetic fields. J. Math. Phys., 38(3):1289-1317, 1997.

[FH10] S. Fournais and B. Helffer. Spectral Methods in Surface Superconductivity, volume 77. Springer Science \& Business Media, 2010.

[FK11] S. Fournais and A. Kachmar. Nucleation of bulk superconductivity close to critical magnetic field. Adv. Math., 226(2):1213-1258, 2011.

[FMP19] S. Fournais, J.P. Miqueu, and X.B. Pan. Concentration behavior and lattice structure of 3D surface superconductivity in the half space. Math. Phys. Anal. Geom., 22(2):12, 2019.

[FPS15] S. Fournais and M. Persson-Sundqvist. Lack of diamagnetism and the Little-Parks effect. Commun. Math. Phys., 337(1):191-224, 2015.

[GP99] T. Giorgi and D. Phillips. The breakdown of superconductivity due to strong fields for the Ginzburg-Landau model. SIAM J. Math. Anal., 30:341-359, 1999.

[HK15] B. Helffer and A. Kachmar. The Ginzburg-Landau functional with vanishing magnetic field. Arch. Ration. Mech. Anal., 218(1):55-122, 2015.

[HK17] B. Helffer and A. Kachmar. Decay of superconductivity away from the magnetic zero set. Calc. Var. Partial Differ. Equ., 56(5):130, 2017.

[HK18] B. Helffer and A. Kachmar. The density of superconductivity in domains with corners. Lett. Math. Phys., 108(9):2169-2187, 2018.

[HK19] B. Helffer and A. Kachmar. Thin domain limit and counterexamples to strong diamagnetism. arXiv:1905.06152, 2019.

[HM01] B. Helffer and A. Morame. Magnetic bottles in connection with superconductivity. J. Funct. Anal., 185(2):604-680, 2001.

[HP03] B. Helffer and X.B. Pan. Upper critical field and location of surface nucleation of superconductivity. Ann. Inst. H.Poincaré Anal. Non Linéaire, 20(1):145-181, 2003.

[Jad01] H.T. Jadallah. The onset of superconductivity in a domain with a corner. J. Math. Phys., 42(9):4101-4121, 2001.

[KP19] A. Kachmar and X.B. Pan. Superconductivity and the Aharonov-Bohm effect. Comptes Rendus Mathematique, 357(2):216-220, 2019.

[KPS19] A. Kachmar and M. Persson-Sundqvist. Counterexample to strong diamagnetism for the magnetic Robin Laplacian. arXiv:1910.12499, 2019.

[LP62] W.A. Little and R.D. Parks. Observation of quantum periodicity in the transition temperature of a superconducting cylinder. Phys. Rev. Lett., 9(1):9-12, 1962. 
[LP99] K. Lu and X.B. Pan. Estimates of the upper critical field for the Ginzburg-Landau equations of superconductivity. Physica D, 127(1):73-104, 1999.

[LP00] K. Lu and X.B. Pan. Gauge invariant eigenvalue problems in $\mathbb{R}^{2}$ and $\mathbb{R}_{+}^{2}$. Trans. Amer. Math. Soc., 352(3):1247-1276, 2000.

[Pan02] X.B. Pan. Upper critical field for superconductors with edges and corners. Calc. Var. Partial Differ. Equ., 14(4):447-482, 2002.

[PK02] X.B. Pan and K.H. Kwek. Schrödinger operators with non-degenerately vanishing magnetic fields in bounded domains. Trans. Amer. Math. Soc., 354(10):4201-4227, 2002.

[Ray09] N. Raymond. Sharp asymptotics for the Neumann Laplacian with variable magnetic field: case of dimension 2. Ann. Henri Poincaré, 10(1):95-122, 2009.

[SJG63] D. Saint-James and P.G. de Gennes. Onset of superconductivity in decreasing fields. Phys. Lett., 7(5):306-308, 1963.

[SS03] E. Sandier and S. Serfaty. The decrease of bulk superconductivity close to the second critical field in the Ginzburg-Landau model. SIAM J. Math. Anal., 34(4):939-956, 2003.

[SS07] E. Sandier and S. Serfaty. Vortices in the Magnetic Ginzburg-Landau Model. Progress in Nonlinear Partial Differential Equations and their Applications, Birkhäuser-Boston, 2007.

Lund University, Department of Mathematics, Lund, Sweden 